\title{
Brote de gastroenteritis aguda en la Región de Antofagasta, Chile. 2010
}

\author{
Janepsy Díaz T., Verónica Solari G., Omar Cáceres C., Javier Mena A., Silvia Baeza P., Ximena Muñoz U., \\ Miguel O'Ryan G., Héctor Galeno A., Aurora Maldonado B. y Nora Mamani M.
}

\section{Outbreaks of acute gastroenteritis in Antofagasta Region. Chile 2010}

Outbreaks of acute gastroenteritis are a public health problem. Norovirus is known as the most common cause (50\%). In Chile, immediate notification allows surveillance of these events. We describe an acute gastroenteritis outbreak that occurred in Antofagasta region, between March and April 2010. An observational study was conducted to perform the outbreak investigation. Local residents who met case definition were included. Stool samples, epidemiological surveys and environmental samples were requested. The outbreak began approximately on March 8, 2010 and lasted until April 28 with 31,036 reported cases (rate 54 per 1000 inhabitants). The most affected age group was between 25 and 44 years, and diarrhea was the main symptom ( $97 \%$ of cases). We determined the presence of norovirus genogroup II in clinical and environmental samples. This outbreak was caused by consumption of raw vegetables from La Chimba, which were watered and contaminated with treated sewage containing low concentration of free residual chlorine. Subsequently, the outbreak spread from person to person in a poor sanitary environment.

Key words: Gastroenteritis, outbreak, norovirus.

Palabras clave: Gastroenteritis aguda, brote, norovirus.

\section{Introducción}

$\mathrm{L}$ a gastroenteritis aguda constituye la primera causa de morbilidad en los países en vías de desarrollo, siendo un problema de salud que origina grandes brotes epidémicos, los que pueden ser causados por diferentes agentes como bacterias, parásitos y virus enteropatógenos, entre los que destacan rotavirus, calicivirus, astrovirus y adenovirus. Se estima que estos virus pueden causar el $80 \%$ de las gastroenteritis agudas ${ }^{1,2}$.

Los calicivirus del tipo norovirus son la causa más común de gastroenteritis epidémica en el mundo y una causa importante de enfermedades transmitidas por alimentos. Datos de Estados Unidos de Norteamérica y países europeos, han demostrado que norovirus es responsable de aproximadamente $50 \%$ de todos los brotes de gastroenteritis reportados (rango: $36-59 \%)^{3}$.

En E.U.A., se estima que aproximadamente 21 millones de cuadros gastroentéricos son atribuibles a norovirus cada año, desde el año 2001, cuando fueron publicadas las recomendaciones más recientes de norovirus ${ }^{4}$. En la mayoría de los brotes que ocurren en países en vías de desarrollo no se puede determinar su presencia debido a las limitaciones en los diagnósticos de laboratorio, clínicos y epidemiológicos ${ }^{5}$.

Este virus puede ser transmitido por los alimentos, el agua y las superficies contaminadas del medio ambiente, así como directamente de persona a persona ${ }^{6-8}$. Los brotes pueden ocurrir en una variedad de entornos institucionales (hogares de ancianos, hospitales y escuelas) e infectar a personas de todas las edades, asociándose con mayor frecuencia a la enfermedad transmitida por los alimentos; no se reporta inmunidad de larga duración ${ }^{9,10}$.

Estos brotes son considerados un importante problema de salud pública, su verdadera magnitud es difícil de estimar y suelen presentarse como casos aislados leves y pasar desapercibidos por los sistemas de notificación y vigilancia $^{11}$. En nuestro medio, los posibles brotes ocasionados por esta enfermedad son detectados por médicos, enfermeras y profesionales de laboratorios clínicos, quienes perciben aumentos inusuales en el número de consultas por una determinada patología e informan de ello a las redes de vigilancia epidemiológica del sistema de salud de Chile $^{12}$. Conocer la magnitud, distribución y los factores de riesgo específicos asociados a un brote de gastroenteritis aguda es un primer paso para lograr su mitigación ${ }^{13}$.

La vigilancia epidemiológica es y ha sido la base para detectar la ocurrencia de brotes epidémicos. El monitoreo de eventos inusuales permite la recolección, análisis e interpretación de la información, con el fin de actuar oportunamente sobre los problemas que suponen un riesgo para la salud de la población, así como evaluar el impacto de la intervención ${ }^{14}$.
Ministerio de Salud de Chile. Departamento de Epidemiología Unidad de Vigilancia (JDT). SEREMI Salud Metropolitana Subdepartamento de Epidemiología (VSG).

Subdepartamento Entorno

Saludable (OCC).

Departamento de Alimentos y Nutrición (SBP).

SEREMI Salud Antofagasta.

Subdepartamento de Epidemiología (JMA)

SEREMI Salud Iquique.

Departamento de Acción Sanitaria (XMU).

Universidad de Chile.

Facultad de Medicina

Instituto de Ciencias Biomédicas Programa de Microbiología y Micología (MOG, NMM) Instituto de Salud Pública. Departamento de Virología (HGA). Departamento de Bacteriología (AMB) .

Fuente de financiamiento: Estudio de brote realizado por Departamento de Epidemiología del MINSAL y la Seremi de Salud de la Región Metropolitana. Chile.

Los autores declaran no tener conflicto de interés.

Recibido: 22 de marzo de 2011 Aceptado: 2 de agosto de 2011

Correspondencia a: Verónica Solari Gallegos veronica.solari@redsalud.gov.cl 
En Chile, todos los brotes, cualquiera sea su agente causal y su magnitud, son de notificación obligatoria inmediata, según lo establece el Reglamento sobre Notificación de Enfermedades Transmisibles de Declaración Obligatoria (Decreto Supremo No 158/2004 del MINSAL) $)^{15}$. Durante el período de verano aumenta la notificación de brotes de gastroenteritis agudas en el país, situación favorecida por las condiciones ambientales y la inadecuada aplicación de las medidas higiénicas sanitarias por parte de la población.

Frente al aumento de casos por gastroenteritis aguda en la Región de Antofagasta, el Ministerio de Salud (MINSAL) envió un Equipo de Respuesta Rápida (ERR) multidisciplinario, conformado por médicos, médicos veterinarios, epidemiólogos, ingenieros, microbiólogos y tecnólogos médicos de la Región Metropolitana, con el objetivo de caracterizar el brote ocurrido durante los meses de marzo y abril de 2010.

\section{Material y Método}

Descripción de la investigación. Se constituyó un ERR para realizar la investigación epidemiológica y se desarrolló un estudio observacional de tipo descriptivo, insertado en una investigación de brote de tipo operacional.

Se definió el brote de gastroenteritis aguda de la Región de Antofagasta como el problema a investigar.

Universo de estudio. La Región de Antofagasta está situada al norte de Chile, y cuenta con una población de 575.268 habitantes; $52 \%$ de sexo masculino (n: 299.473) y el resto de sexo femenino (n: 275.795), distribuida en tres provincias: Antofagasta, El Loa y Tocopilla ${ }^{16}$.

El universo de estudio fueron todos los casos que consultaron en un establecimiento de salud de la región, durante los meses de marzo y abril de 2010, que cumplieron con la definición de caso establecida y/o tenían nexo epidemiológico.

Definición de caso. Todo paciente que consultó entre el 8 de marzo y el 28 de abril de 2010 en un servicio de urgencia, público o privado, de la Región de Antofagasta, que presentase diarrea y dolor abdominal, más uno de los siguientes síntomas; vómitos, náuseas o fiebre mayor o igual a $38^{\circ} \mathrm{C}$.

Análisis de la información. Se realizó una encuesta epidemiológica domiciliaria a 103 de los casos presentados. La selección de los casos se realizó a través de un muestreo aleatorio (utilizando una tabla de números aleatorios) mediante el programa Epinfo versión 6,2. La encuesta aplicada contenía variables relacionadas con: identificación del paciente (nombre, dirección, edad, teléfono, actividad) y variables epidemiológicas como: agente etiológico aislado en deposición, fecha de los primeros síntomas, síntomas y signos clínicos, frecuencia de la diarrea, duración, lugar de atención médica, antecedentes de familiares u otras personas enfermas, referencia de lugar de abastecimiento y consumo de verduras y hortalizas crudas, fuente de agua potable u otra fuente embotellada de agua, problemas con la recolección de basuras domiciliarias y aseo de la vivienda.

Se confeccionó una base de datos en la hoja de cálculo Excel 2003 y se realizó un análisis descriptivo de los datos y cruce de variables mediante el programa estadístico EpiInfo 2004.

Se consideró que el brote estaba controlado cuando el número de consultas por semana epidemiológica alcanzó el mismo nivel que antes de su inicio.

Estudio de las muestras clínicas. Se tomaron muestras de deposiciones a 932 casos $(3,0 \%)$, que cumplieron con la definición de caso. La selección de los casos se realizó de forma dinámica, según se fueron presentando, debido a la disponibilidad de recursos humanos y materiales en los establecimientos de salud. A las muestras tomadas se les realizó estudio viral, bacteriano y parasitológico. Los análisis fueron efectuados en los laboratorios del Instituto de Salud Pública (ISP) y en el laboratorio del Instituto de Ciencias Biomédicas de la Facultad de Medicina de la Universidad de Chile, en un trabajo colaborativo entre ambas instituciones y el Ministerio de Salud.

Cada frasco se rotuló con los datos del paciente, fecha de toma de muestra, edad o fecha de nacimiento y fecha de primeros síntomas, cuando se disponía de este último dato. Posteriormente, se trasladó en una caja con aislamiento térmico para su conservación, a la Región Metropolitana.

Para el estudio viral se tomó una muestra de deposición fresca, con menos de una hora de emitida, no mezclada con orina y recolectada en un recipiente limpio y seco. Dicha muestra se conservó en refrigeración a $4^{\circ} \mathrm{C}$, por un máximo de dos días. En el caso que se requirió guardar la muestra por más tiempo, se conservó a $-20{ }^{\circ} \mathrm{C}$. El diagnóstico de norovirus se realizó en el Instituto de Salud Pública en forma paralela con el Instituto de Ciencias Biomédicas de la Facultad de Medicina de la Universidad de Chile mediante la detección del antígeno de norovirus I/II en una suspensión de la muestra fecal (método comercial RIDASCEEN Norovirus $3^{\text {a }}$ Generation, R-Biofarm, Alemania) y mediante la detección de genoma de norovirus I y II en un purificado de ácido ribonucleico (ARN) extraído de la muestra fecal, según el procedimiento transcripción reversa ligada a reacción de polimerasa en cadena (TRRPC) de tiempo real, descrito por Burkhardt W. y cols. ${ }^{18}$, que incluye partidores para los dos genogrupos de norovirus más comúnmente detectados en brotes de diarrea en el mundo, sondas genéticas tipo Taqman de elevada 
especificidad para norovirus genogrupo I y genogrupo II, sistema de control interno para verificación de inhibidores en cada tubo de reacción. Los extractos de ARN positivos para norovirus fueron enviados a secuenciación genética.

Para estudio bacteriano se tomó un coprocultivo en el momento de la consulta en el Servicio de Urgencia. Este coprocultivo se conservó en medio de transporte Cary Blair y AMIES, hasta su análisis, y se le determinó a cada uno, presencia de Salmonella sp, Shigella sp, Escherichia coli diarreogénica, Yersinia enterocolitica, Listeria monocytogenes, Vibrio parahaemolyticus, Aeromonas sp y Campylobacter jejuni.

Las cepas de Salmonella sp y Shigella sp aisladas en el laboratorio del hospital de Antofagasta fueron confirmadas y serotipificadas por el ISP.

El estudio parasitológico se realizó sólo en el ISP. La muestra de deposición fresca se mezcló con el líquido fijador para su transporte y se conservó a temperatura ambiente. Se realizó sólo observación microscópica. No fue posible hacer concentración por el escaso volumen de muestra. Se realizó técnica inmunocromatográfica para Cryptosporidium sp y Giardia sp.

Estudio de muestras ambientales. Se obtuvieron muestras de agua potable y agua servida tratada. La toma de muestra se realizó filtrando 20 litros mediante la técnica de ultracentrifugación según lo descrito por Polaczyk y $\operatorname{cols}^{17}$. Estas muestras fueron procesadas en el laboratorio de la Universidad de Chile y en el del ISP. Fueron sometidas a extracción de las partículas virales por medio de ultrafiltración y ultracentrifugación y posterior detección molecular, utilizando el método descrito por Burkhard III y cols ${ }^{18}$, RPC para los genogrupos I y II de norovirus.

Las muestras positivas a la presencia de norovirus fueron sometidas a secuenciación molecular según protocolo de la Sección Genética Molecular del ISP ${ }^{19}$, con el objeto de establecer la relación entre los virus aislados en las muestras clínicas y los aislados en muestras de agua.

Se tomaron muestras de alimentos de riesgo consumidos por los pacientes encuestados, tales como ensaladas surtidas, mariscos crudos y hortalizas de consumo crudo, las que fueron procesadas en el ISP y en el Laboratorio Ambiental Regional de Antofagasta.

\section{Resultados}

Entre el 8 de marzo y el 28 de abril de 2010, consultaron en la Región de Antofagasta 31.036 casos de gastroenteritis aguda (tasa 54 por 1.000 habitantes), los que cumplían con la definición de caso. Se estimó que el brote comenzó en los primeros días de marzo (semana epidemiológica 9). La curva epidémica reflejó una fuente común continua, con transmisión de persona a persona,

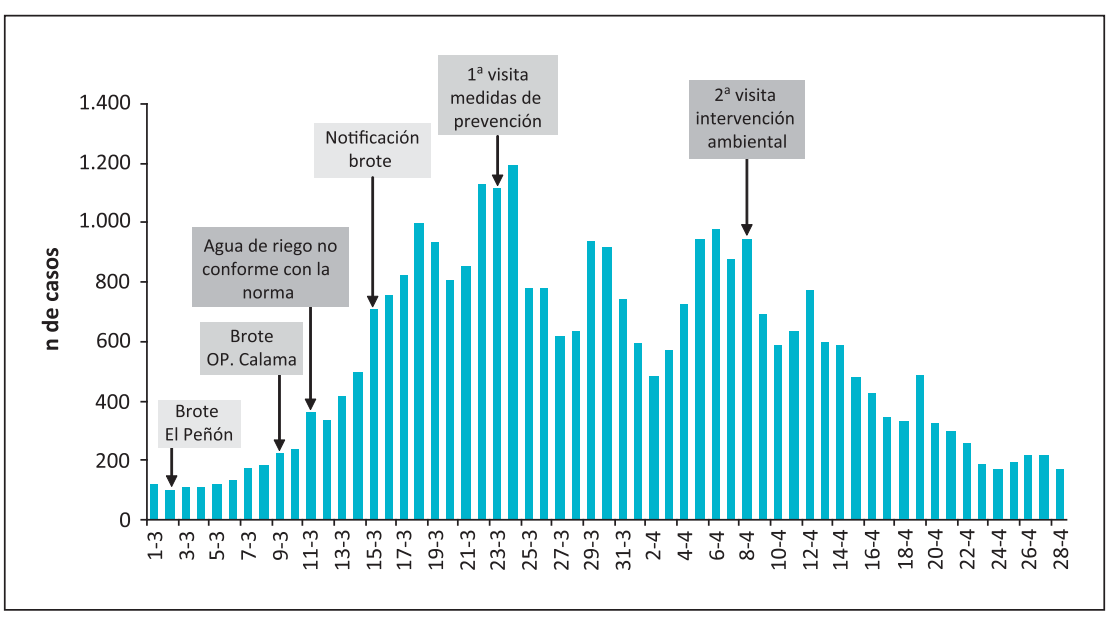

Figura 1. Consultas de gastroenteritis aguda según fecha de consulta. Región de Antofagasta, marzo-abril de 2010.

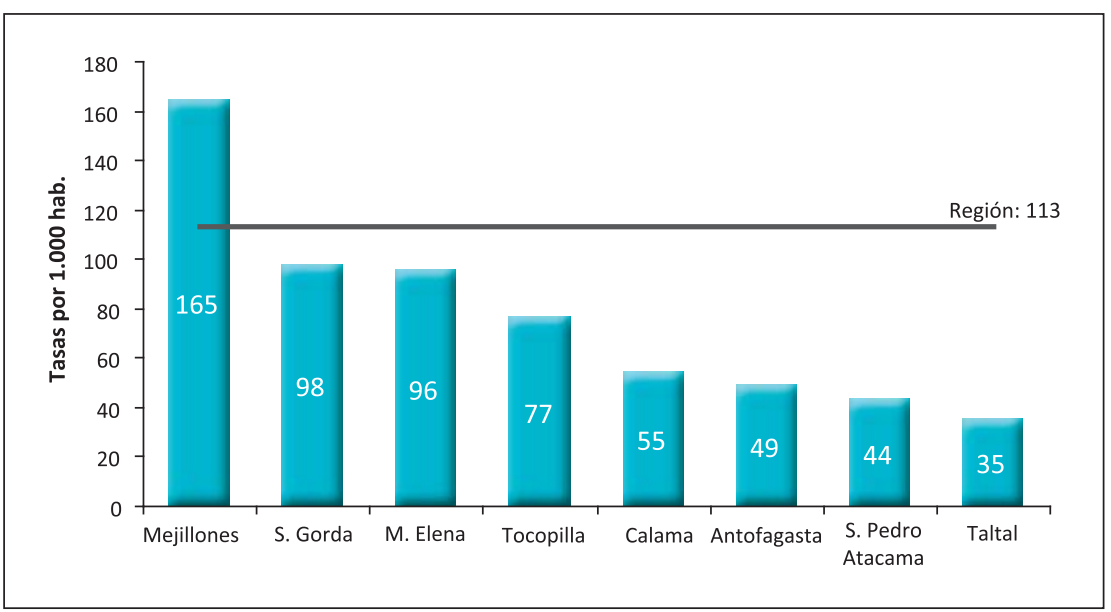

Figura 2. Tasa de gastroenteritis aguda por comuna. Región de Antofagasta marzo-abril de 2010.

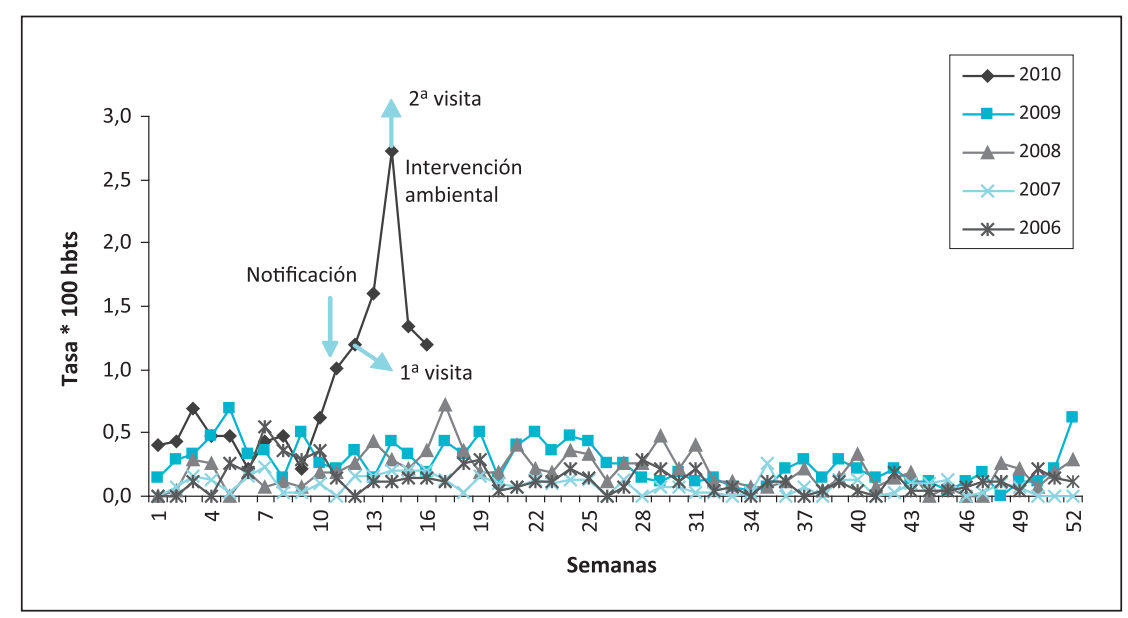

Figura 3. Vigilancia centinela de diarrea, Consultorio Juan Pablo II. Antofagasta 2010. 
Tabla 1. Resultados de muestras clínicas para estudio virológico. ISP Chile 2010

\begin{tabular}{lrrrrrrrrr} 
Centros & \multicolumn{3}{c}{ Norovirus gg- I } & \multicolumn{3}{c}{ Norovirus gg-II } & \multicolumn{3}{c}{ Total norovirus } \\
& $n$ & + & $\%+$ & $n$ & + & $\%+$ & $n$ & + & $\%+$ \\
H. Antofagasta & 59 & 2 & 3,4 & 59 & 9 & 15,3 & 59 & 11 & 18,6 \\
CAN & 48 & 0 & 0,0 & 48 & 8 & 16,7 & 48 & 8 & 16,7 \\
CJPII & 45 & 2 & 4,4 & 45 & 2 & 4,4 & 45 & 4 & 8,9 \\
Hosp. Calama & 26 & 0 & 0,0 & 26 & 0 & 0,0 & 26 & 0 & 0,0 \\
Hosp. Mejillones & 20 & 0 & 0,0 & 20 & 0 & 0,0 & 20 & 0 & 0,0 \\
Hosp. Tal Tal & 5 & 1 & 20,0 & 5 & 1 & 20,0 & 5 & 2 & 40,0 \\
Total & 203 & 5 & 2,5 & 203 & 20 & 9,9 & 203 & 25 & 12,3 \\
\hline
\end{tabular}

Fuente: Laboratorio de Virología. ISP. CAN: CJPII: Consultorio Juan Pablo II.
En el Laboratorio de Bacteriología del ISP se estudiaron 712 muestras, en $63,7 \%$ se aisló E. coli diarreogénica (454/712), Shigella sp en 5,2\% (37/712) y 1,5\% (11/712) Vibrio cholerae no $\mathrm{O} 1$ no O139. En el 29,5\% restante (210 muestras) no se aisló agente bacteriano.

En el Laboratorio de parasitología se estudiaron 31 muestras. En 16\% (5 muestras), se pudo aislar Giardia lamblia y en una muestra Cryptosporidium sp.

Se realizó encuestas domiciliarias a los casos confirmados con calicivirus, ECET, y $V$. cholerae no $\mathrm{O} 1$ no O139, y se determinó como factor de riesgo común el abastecimiento y consumo de verduras y platos listos para el consumo en mercados y ferias de la zona norte de la comuna de Antofagasta (Feria Las Pulgas, Feria Juan Pablo II). El 100\% de los casos encuestados correspondió a pacientes involucrados en brotes intra-familiares.

Resultados de los análisis en muestras ambientales. Las muestras de agua potable tomadas por el sistema de vigilancia en los domicilios particulares de la ciudad de Antofagasta resultaron con ausencia de norovirus y negativas a coliformes totales y fecales. Las muestras de agua tomadas en la cordillera, agua desalada, mezclada y de pozo, tomadas por el ISP para estudio de parásitos resultaron negativas a Cryptosporidium sp y a Giardia lamblia. En una muestra se observó un quiste de Entamoeba histolytica.

Se detectó la presencia de genoma de calicivirus y rotavirus en agua de la planta de tratamiento de aguas servidas de la ciudad de Antofagasta. La muestra positiva para calicivirus fue caracterizada en la Universidad de Chile y correspondió a norovirus genogrupo II. Asimismo, las muestras de la planta de tratamiento de agua servida, tomadas en el afluente y efluente de la planta, y procesadas por el ISP, resultaron con presencia de norovirus genogrupo II.

El análisis genético de estos cinco norovirus demostró que correspondían a genogrupo II con $94-98 \%$ de similitud.

Muestras de alimentos. En abril de 2010 se analizaron en el ISP 55 muestras de ensaladas surtidas y mariscos crudos, detectándose que cuatro muestras de ensaladas surtidas presentaron no conformidad con el Reglamento Sanitario de los Alimentos (tres por presencia de Staphylococcus aureus y una por E. coli diarreogénica). No se estudiaron norovirus en muestras de alimentos.

\section{Discusión}

La investigación epidemiológica del EER en terreno permitió establecer la magnitud y el origen del brote de gastroenteritis aguda ocurrido en la Región de Antofagasta en los meses de marzo y abril del año 2010. Entre el 2 y 
11 de marzo de 2010 se detectó retrospectivamente que se habían presentado dos brotes institucionales con similares características; uno en un campamento minero de Calama y el otro, en funcionarios de la Oficina Provincial de la SEREMI de Calama. Ambos brotes fueron considerados como una enfermedad transmitida por alimentos-ETA. En la literatura médica se menciona la implicancia de norovirus en los brotes de gastroenteritis debido a la transmisión alimentaria, produciéndose la manifestación en el largo plazo en los servicios de atención ${ }^{20,21}$.

Por otra parte, muestras ambientales, tomadas a principios del año 2010, por la SEREMI de Salud de Antofagasta, detectaron la presencia de coliformes fecales por sobre 1.600 NMP /100 ml en el agua utilizada para el riego de hortalizas que se consumen crudas y que crecen a ras de suelo en el sector agrícola La Chimba. Asimismo, las mediciones de cloro libre residual y concentraciones de coliformes fecales en aguas servidas tratadas, mostraron que a los predios el agua llegaba con bajas concentraciones de cloro libre $(<0,6 \mathrm{mg} / \mathrm{L}$ según lo establecido por Resolución No 550 del 26.03.1991 del Servicio de Salud Antofagasta). Todo esto, sumado a las graves deficiencias sanitarias en los procesos de manejo y almacenamiento del agua servida tratada, y usada para el riego de hortalizas que crecen a ras de suelo y se consumen crudas, tales como lechugas, perejil, cilantro, albahaca, rábanos, entre otros, en el sector La Chimba, constituyeron los factores de riesgos ambientales a los que se mantuvo expuesta la población durante el período de estudio.

Se constató además, que los alimentos cultivados en el sector La Chimba se expendían en dos ferias ubicadas en sector norte de Antofagasta (Feria Las Pulgas y Feria Juan Pablo II), las que se encontraban muy cercanas a los domicilios de los casos encuestados. También se expendían frutas frescas, mariscos y pescados crudos preparados, todos alimentos procesados por los mismos feriantes, que también habían enfermado durante el período de estudio, transformándose en agentes transmisores de norovirus. Asimismo, la Vega Central ubicada en el centro de la ciudad, formaba parte de comercialización y distribución mayorista de verduras y hortalizas para las otras comunas de la región (Calama, Mejillones y Tocopilla), las que también procedían en gran parte del sector La Chimba. Lo planteado refleja el incumplimiento con los parámetros establecidos en la Norma Chilena $\mathrm{N}^{\circ}$ $1333^{22}$, que regula la reutilización de las aguas servidas para el riego de hortalizas de consumo crudo.

Esta situación puntual se debió a la dificultad de proveerse de hipoclorito de sodio desde la planta ubicada en la ciudad de Talcahuano, producto del deterioro sufrido en sus instalaciones a raíz del terremoto acaecido el 27 de febrero de 2010 en el país.

Los resultados obtenidos en la investigación realizada por el ERR, asociados a la presencia de norovirus geno- grupo II en aguas servidas tratadas con las que fueron regadas las hortalizas de consumo crudo y el resultado del análisis genético de las muestras clínicas y ambientales, que demostró correspondencia a norovirus genogrupo II en $94-98 \%$ de similitud, nos permitió identificarlo como el agente principal causal de este gran brote de gastroenteritis aguda. Desde 2001, el genogrupo II.4 se ha asociado con la mayoría de los brotes de gastroenteritis virales en todo el mundo ${ }^{23}$. Como se desprende de la bibliografía revisada, en el año 2001 el CDC publicó las recomendaciones sobre los avances importantes que se han realizado en la epidemiología, inmunología, métodos de diagnóstico y control de la infección por norovirus ${ }^{24}$, lo que nos ha permitido dar un paso importante en la salud pública para la prevención y el control de la enfermedad causada por este virus.

Este agente causal, que tiene una alta transmisión de persona a persona, explica la extensión geográfica y magnitud del brote en la Región de Antofagasta. En dicha región se presentan habitualmente pequeños brotes asociados a los agentes bacterianos identificados en nuestro estudio. Sin embargo, estos han tenido una presentación autolimitada en número de personas afectadas. Se menciona en la literatura científica que hasta $30 \%$ de las infecciones por norovirus son asintomáticas, y las personas asintomáticas pueden diseminar el virus, aunque a títulos más bajos que las personas sintomáticas ${ }^{25,26}$. Aproximadamente sólo $10 \%$ de las personas afectadas con gastroenteritis por norovirus busca atención médica, que podría incluir la hospitalización y el tratamiento de la deshidratación con la terapia de líquidos por vía oral o intravenosa $\mathrm{s}^{21,27,28}$.

Aunque los síntomas pueden ser graves, por lo general se resuelven sin tratamiento después de 1 a 3 días en personas previamente sanas. Sin embargo, cursos más prolongados de la enfermedad, que duran 4 a 6 días pueden ocurrir, sobre todo entre los niños pequeños, personas de edad avanzada y pacientes hospitalizados ${ }^{29,30}$. Nuestro estudio reflejó que la población mayormente afectada correspondió a adultos jóvenes sanos, con sintomatología menos grave, lo que justifica que no hubo casos hospitalizados y ni fallecidos.

La literatura científica plantea que estos brotes se producen durante todo el año, aunque puede existir un patrón estacional de mayor actividad durante los meses de invierno y que estos aumentos periódicos en los brotes de norovirus tienden a ocurrir en asociación con la aparición de nuevas cepas GII. $4^{31,32}$. Sin embargo, en Chile se ha observado con mayor frecuencia la aparición de estos brotes durante el verano ${ }^{33}$.

Varias rutas de transmisión pueden ocurrir en la historia natural de un brote; en nuestro estudio se concluyó que el brote se relacionó con una fuente puntual, debida a una exposición de alimentos contaminados, fundamentalmente por norovirus, asociado con una dispersión 
secundaria de la infección por la transmisión de persona a persona dentro de la comunidad. Con este análisis el ERR implementó medidas higiénico epidemiológicas y ambientales, con el fin de cortar la cadena de transmisión, la cual estuvo dirigida fundamentalmente a no usar el agua servida tratada para riego de hortalizas de consumo crudo, a partir del 8 de abril de 2010; además de reiterar a la población las medidas de prevención de enfermedades entéricas mediante una campaña comunicacional, enfatizando en el lavado de manos, lavado de verduras y frutas, incluso las empaquetadas, mantener limpios y desinfectados los servicios higiénicos, cocinas y sus superficies, beber siempre agua potable y si no se dispusiera de ella hervirla, además de adquirir y consumir alimentos en lugares autorizados, entre otras.

Con estas medidas implementadas comenzó el descenso del brote desde el 13 de abril de 2010, lográndose de esta manera su control en la Región de Antofagasta.

Los autores recomendamos la revisión de la normativa que regula la reutilización de las aguas servidas para el riego de hortalizas de consumo crudo, de manera de evitar la ocurrencia de este tipo de eventos en el país. Además de contar con la disponibilidad de técnicas de laboratorio en la red de salud pública para identificación de virus entéricos en matrices ambientales (agua y alimentos), situación que dificultó obtener resultados oportunos en la investigación del brote.

\section{Resumen}

Antecedentes: Los brotes por gastroenteritis aguda constituyen un problema de salud pública. Se conoce al norovirus como la causa más común $(50 \%)$. En Chile, la vigilancia de estos eventos, se establece mediante la notificación inmediata. Objetivo: Investigar y caracterizar el brote de gastroenteritis aguda ocurrido en la Región de Antofagasta, durante los meses de marzo y abril de 2010. Método: Se efectuó un estudio observacional descriptivo para realizar la investigación de brote. Se incluyó a residentes de la región que cumplían con la definición de caso. Se solicitó muestras de deposición, encuesta epidemiológica y muestras ambientales. $R e$ sultados: Se estimó que el brote comenzó el 8 de marzo de 2010 y duró hasta el 28 de abril del mismo año; se notificaron 31.036 casos (tasa 54 por 1.000 habitantes). El grupo de 25 y 44 años de edad fue el más afectado y la diarrea fue el síntoma predominante $(97 \%$ de los casos). Se determinó la presencia de norovirus genogrupo II en muestras clínicas y ambientales. Conclusiones: El brote se originó por el consumo crudo de hortalizas que provenían del sector La Chimba, las que fueron regadas y contaminadas con agua servida tratada que contenía baja concentración de cloro libre residual y posteriormente se propagó por transmisión persona-persona, en un ambiente sanitario deficiente.

\section{Referencias}

1.- Mead P S, Slutsker L, Dietz V, McCaig L F, Bresee J S, Shapiro C, et al. Food-relates illness and death in the United States. Emerg Infect Dis 1999; 5: 607-25.

2.- Marie-Cardine A, Gourlain K, Mouterde O, Castignolles N, Hellot M F, Mallet E, et al. Epidemiology of acute viral gastroenteritis in children hospitalized in Rouen, France. Clin Infect Dis 2002; 34: 1170-8.

3.- Patel M M, Hall A J, Vinje J, Parashar U D. Noroviruses: a comprehensive review. J Clin Virol 2009; 44: 1-8.

4.- Scallan E, Hoekstra R M, Angulo F J, Tauxe R V, Widdowson M A, Roy S L, et al. Foodborne illness acquired in the United States major pathogens. Emerg Infect Dis 2011; 17: 7-15.

5.- Kaplan J E, Feldman R, Campbell D S, Lookabaugh C, Gary G W. The frequency of a Norwalk-like pattern of illness in outbreaks of acute gastroenteritis. Am J Public Health 1982; 72: 1329-32.

6.- Doyle T J, Stark L, Hammond R, Hopkins R S. Outbreaks of noroviral gastroenteritis in Florida, 2006-2007. Epidemiol Infect 2009; 137: 617-25.

7.- Yen C, Wikswo M, Lopman B, Hall A.
Temporal trends in norovirus activity United States, January 2007-April 2010 [Presentation]. $4^{\text {th }}$ International Conference on Caliciviruses, Santa Cruz, Chile; October 16-19, 2010.

8.- Chin J. El Control de las Enfermedades Transmisibles, 1 edición 2001: 310-15.

9.- Blanton L H, Adams S M, Beard R S, Wei G, Bulens S N, Widdowson M A, et al. Molecular and epidemiologic trends of caliciviruses associated with outbreaks of acute gastroenteritis in the United States, 2000-2004. J Infect Dis 2006; 193: 413-21.

10.- Fankhauser R L, Monroe S S, Noel J S, Humphrey C D, Bresee J S, Parashar U D, et al. Epidemiologic and molecular trends of "Norwalk-like viruses" associated with outbreaks of gastroenteritis in the United States. $\mathrm{J}$ Infect Dis 2002; 186: 1-7.

11.- Tekinşen K K, Özdemir Z. Prevalence of foodborne pathogens in Turkish Van otlu (Herb) cheese. Food Cont 2006; 17: 707-11. doi:10.1016/j.foodcont.2005.04.007. doi:10.1016/j.jfoodcont.2005.04.007. [Cross Ref].

12.- Reingold A. Investigaciones de brotes - Una perspectiva. Boletín Epidemiológico: OPS; Junio 2000; 21 (2): 2-7.

13.- Jones T F, McMillian M B, Scallan E,
Frenzen P D, Cronquist A B, Thomas S, et al. A population-based estimate of the substantial burden of diarrhoeal disease in the United States; FoodNet, 1996-2003. Epidemiol Infect 2007; 135: 293-301.

14.- OPS. Vigilancia Epidemiológica. En: Principios de Epidemiología para el Control de Enfermedades. Unidad de Epidemiología. Programa ampliado de libros de texto de la OMS. 2000.

15.- Ministerio de Salud de Chile. Reglamento sobre Notificación de Enfermedades Transmisibles de Declaración Obligatoria. Decreto Supremo No 158. Departamento Asesoría Jurídica: Ministerio de Salud. Chile. 22 de octubre de 2004. (Disponible en: http://epi.minsal.cl/epi/ $\mathrm{html} /$ frames/frame1.htm)

16.- Instituto Nacional de Estadística de Chile. Censo Poblacional. 2002.

17.- Polaczyk A L, Narayanan J, Cromeans T L, Hahn D, Roberts J M, Amburgey J E, et al. Ultrafiltration-based techiques for rapid and simultaneous concentration of multiple microbe classes from $100 \mathrm{~L}$ tap water samples. J Microbiol Methods 2008; 73: 92-9.

18.- Burkhardt III W, Pryor J. A real time RT-PCR protocol for the simultaneous detection of 
norovirus and enterovirus. Laboratory Bulletin $\mathrm{N}^{\circ} 4369$.

19.- Beuret C, Kohler D, Baumgarten A, Lüthi T M. Norwalk-like sequences in mineral waters: oneyear monitoring of three brans. Appl Environ Microbiol 2002; 68 (4): 1925-31.

20.- Rosenthal N A, Lee L E, Vermeulen B A, Hedberg K, Keene W E, Widdowson M A, et al Epidemiological and genetic characteristics of norovirus outbreaks in long-term care facilities, 2003-2006. Epidemiol Infect 2011; 139: 286-94.

21.- Widdowson M A, Sulka A, Bulens S N, Beard R S, Chaves S S, Hammond R, et al. Norovirus and foodborne disease, United States, 1991-2000. Emerg Infect Dis 2005; 11: 95-102.

22.- Norma Chilena Oficial NCh 1333 Of 78. Decreto $\mathrm{N}^{\circ}$ 867/78: 20 pp.

23.- Siebenga J J, Vennema H, Zheng D P, Vinjé J, Lee B E, Pang X L, et al. Norovirus illness is a global problem: emergence and spread of Norovirus GII.4 Variants, 2001-2007. J Infect Dis 2009; 200: 802-12.

24.- CDC. "Norwalk-like viruses". Public health consequences and outbreak management. MMWR Morb Mortal Wkly Rep 2001; 50 (No.
RR-9).

25.- Atmar R L, Opekun A R, Gilger M A, Estes M K, Crawford S E, Neill F H, et al. Norwalk virus shedding after experimental human infection. Emerg Infect Dis 2008; 14 : 1553-7.

26.- Phillips G, Lopman B, Tam CC, IturrizaGomara M, Brown D, Gray J. Diagnosing norovirus-associated infectious intestinal disease using viral load. BMC Infect Dis 2009; 9: 63.

27.- Phillips G, Tam C C, Conti S, Rodrígues L C, Brown D, Iturriza-Gomara M, et al. Community incidence of norovirus-associated infectious intestinal disease in England: improved estimates using viral load for norovirus diagnosis. Am J Epidemiol 2010; 171: 1014-22.

28.- de Wit M A, Koopmans M P, Kortbeek L M, Wannet W J, Vinjé J, van Leusden F, et al. Sensor, a population-based cohort study on gastroenteritis in the Netherlands: incidence and etiology. Am J Epidemiol 2001; 154: 666-74.

29.- Rockx B, De Wit M, Vennema H, Vinjé J, De
Bruin E, Van Duynhoven Y, et al. Natural history of human calicivirus infection: a prospective cohort study. Clin Infect Dis 2002; 35: 246-53.

30.- Lopman B A, Reacher M H, Vipond I B, Sarangi J, Brown D W. Clinical manifestation of norovirus gastroenteritis in health care settings. Clin Infect Dis 2004; 39: 318-24.

31.- Cannon J L, Lindesmith L C, Donaldson E F, Saxe L, Baric R S, Vinje J. Herd immunity to GII.4 noroviruses is supported by outbreak patient sera. J Virol 2009; 83: 5363-74.

32.- Zheng D P, Widdowson M A, Glass R I, Vinje J. Molecular epidemiology of genogroup II-genotype 4 noroviruses in the United States between 1994 and 2006. J Clin Microbiol 2010; 48: 168-77.

33.- Solari V, Mamani N, Vidal R, Prado V, O'Ryan M. Brotes de gastroenteritis por calicivirus humanos; Relevancia creciente en la Región Metropolitana. Libro de Resúmenes XX Congreso Chileno de Infectología, Hotel Carrera, La Serena, Chile. Noviembre 5-8 de 2003. Abstract CO-31 pág 63. 